FISIOTERAPEUȚA NA ATENÇÃO PRIMARIA EM SAÚDE NO CONTTEXTO

DAS PREVENÇÕES DE QUEDAS EM IDOSOS:

\title{
UMA REVISÃO INTEGRATIVA
}

Rutelene Oliveira Bailosa

Francisco Carlos Santos Cerqueira 
FISIOTERAPEUȚA NA ATENÇÃO PRIMARIA EM SAÚDE NO CONTTEXTO

DAS PREVENÇÕES DE QUEDAS EM IDOSOS:

\section{UMA REVISÃO INTEGRATIVA}

Rutelene Oliveira Bailosa

Francisco Carlos Santos Cerqueira 
2020 by Editora e-Publicar

Copyright $(C)$ Editora e-Publicar

Copyright do Texto (C) 2020 Os autores

Copyright da Edição (C) 2020 Editora e-Publicar

Direitos para esta edição cedidos à Editora e-Publicar pelos autores.

\author{
Editora Chefe \\ Patrícia Gonçalves de Freitas \\ Editor \\ Roger Goulart Mello \\ Diagramação \\ Roger Goulart Mello \\ Projeto gráfico e Edição de Arte \\ Patrícia Gonçalves de Freitas \\ Revisão \\ Os Autores
}

Todo o conteúdo dos artigos, dados, informações e correções são de responsabilidade exclusiva dos autores. O download e compartilhamento da obra são permitidos desde que os créditos sejam devidamente atribuídos aos autores. É vedada a realização de alterações na obra, assim como sua utilização para fins comerciais.

A Editora e-Publicar não se responsabiliza por eventuais mudanças ocorridas nos endereços convencionais ou eletrônicos citados nesta obra.

\title{
Conselho Editorial
}

Dr $^{\mathrm{a}}$ Cristiana Barcelos da Silva - Universidade Estadual do Norte Fluminense Darcy Ribeiro

Dr $^{\mathrm{a}}$ Elis Regina Barbosa Angelo - Pontifícia Universidade Católica de São Paulo

Dr. Rafael Leal da Silva - Universidade Federal do Rio Grande do Norte

Dr. Fábio Pereira Cerdera - Universidade Federal Rural do Rio de Janeiro

Dr $^{\mathrm{a}}$ Danyelle Andrade Mota - Universidade Tiradentes

Me. Doutorando Mateus Dias Antunes - Universidade de São Paulo

Me. Doutorando Diogo Luiz Lima Augusto - Pontifícia Universidade Católica do Rio de Janeiro

Me. Doutorando Francisco Oricelio da Silva Brindeiro - Universidade Estadual do Ceará

$\mathrm{M}^{\mathrm{a}}$ Doutoranda Bianca Gabriely Ferreira Silva - Universidade Federal de Pernambuco

$\mathrm{M}^{\mathrm{a}}$ Doutoranda Andréa Cristina Marques de Araújo - Universidade Fernando Pessoa

Me. Doutorando Milson dos Santos Barbosa - Universidade Tiradentes

$\mathrm{M}^{\mathrm{a}}$ Doutoranda Jucilene Oliveira de Sousa - Universidade Estadual de Campinas

$\mathrm{M}^{\mathrm{a}}$ Doutoranda Luana Lima Guimarães - Universidade Federal do Ceará

$\mathrm{M}^{\mathrm{a}}$ Cristiane Elisa Ribas Batista - Universidade Federal de Santa Catarina 
$\mathrm{M}^{\mathrm{a}}$ AndrelizeSchabo Ferreira de Assis - Universidade Federal de Rondônia

Me.DanielOrdane da Costa Vale - Pontifícia Universidade Católica de Minas Gerais

Me.Glaucio Martins da Silva Bandeira - Universidade Federal Fluminense

Me. Jose Henrique de Lacerda Furtado - Instituto Federal do Rio de Janeiro

Ma Luma Mirely de Souza Brandão - Universidade Tiradentes

Dr ${ }^{\mathrm{a}}$. Rita Rodrigues de Souza - Universidade Estadual Paulista

Dr. Helio Fernando Lobo Nogueira da Gama - Universidade Estadual De Santa Cruz

Dr. Willian Douglas Guilherme - Universidade Federal do Tocantins

Dra ${ }^{\mathrm{a}}$. Naiola Paiva de Miranda - Universidade Federal do Ceará

Dr $^{\mathrm{a}}$. Dayanne Tomaz Casimiro da Silva - UFPE - Universidade Federal de Pernambuco

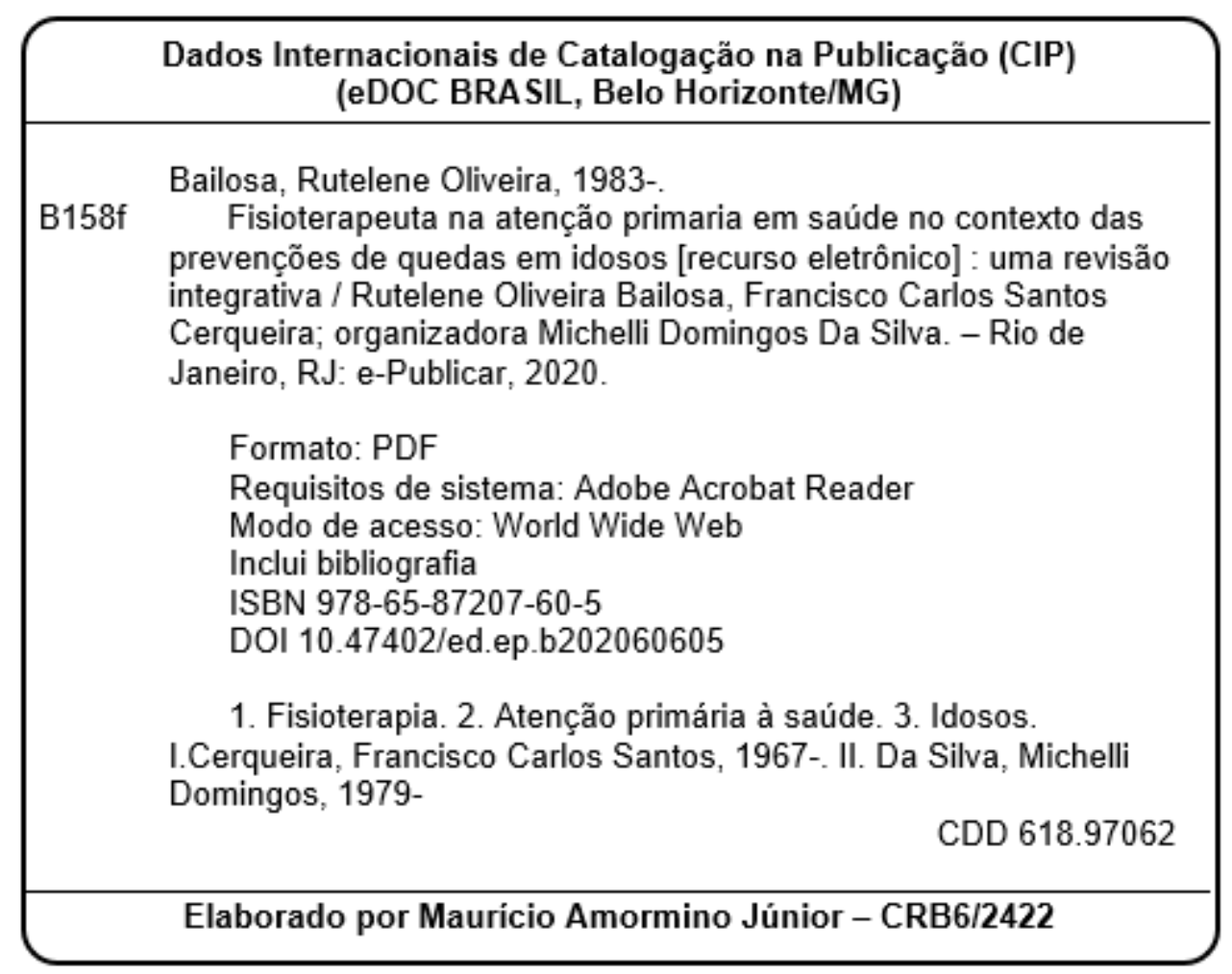

Editora e-Publicar

Rio de Janeiro - RJ - Brasil

contato@editorapublicar.com.br

www.editorapublicar.com.br 


\section{APRESENTAÇÃO}

É com imensa satisfação que apresentamos a obra "Fisioterapeuta na atenção primária em saúde no contexto das prevenções de quedas em idosos: uma revisão integrativa" elaborada por Rutelene Oliveira Bailosa, Francisco Carlos Santos Cerqueira e organizada por Michelli Domingos da Silva.

Desejamos a todos uma excelente leitura!

Patrícia Gonçalves de Freitas

Roger Goulart Mello

Editora e-Publicar 


\section{RESUMO}

Introdução: A Organização das Nações Unidas, estima-se que a população mundial de idosos passará de 841 milhões para 2 bilhões até 2050. No Brasil, informações colhidas através do sistema de informações do Instituto Brasileiro de Geografia e Estatística (IBGE) assinalam que em 2060 o percentual da população com 65 anos ou mais chegará a 25,5\% (58,2 milhões de idosos), enquanto em 2018 essa proporção era de 9,2\% (19,2 milhões). Objetivo: Este estudo pretende descrever a forma de atuação do fisioterapeuta na atenção básica em saúde, bem como refletir sobre a prevenção de quedas em idosos no contexto da atenção primária em saúde. Metodologia: Trata-se de uma pesquisa de cunho exploratório, do tipo revisão integrativa da literatura. Literatura Latino Americana e do Caribe em Ciências da Saúde (LILACS), Scientific Electronic Library Online (SCIELO), Biblioteca Virtual em Saúde Enfermagem Brasil (BDENF), PUBMED. Resultados: Foram analisados 89 artigos publicados entre 2014 a 2020. Com a estratégia de cruzamento: atenção primária em saúde; fisioterapia; queda; idosos. Após uma seleção criteriosa dos resumos, foram selecionados 10 estudos referentes ao tema do projeto.O quadro acima mostra a importância do fisioterapeuta na prevenção das quedas em idosos. Além disso, os autores citados no quadro acima concordam sobre a importância das atividades física essenciais na terceira idade. E ressalta a importância de tratamento adequado em qualquer fase da vida, porém os idosos tem uma relevância não só de tratamento, mas também de prevenção e intervenção, o que ajuda na melhora da qualidade de vida. Conclusão: $O$ tratamento fisioterapêutico pode ser necessário em qualquer fase da vida, porém os idosos tem uma relevância não só de clínico, mas também de prevenção e intervenção, o que ajuda na melhora da qualidade de vida. Viabilizar a saúde junto aos idosos deve tornar-se um habito constante, por isso, é de suma importância o acompanhamento de um profissional qualificado atuando na terceira idade, promovendo praticas preventivas de exercícios físicos.

Palavra - chave: Atenção Primária em Saúde; Fisioterapia; Idoso;Queda. 


\section{INTRODUÇÃO}

A Organização das Nações Unidas, estima-se que a população mundial de idosos passará de 841 milhões para 2 bilhões até 2050. No Brasil, informações colhidas através do sistema de informações do Instituto Brasileiro de Geografia e Estatística (IBGE) assinalam que em 2060 o percentual da população com 65 anos ou mais chegará a 25,5\% (58,2 milhões de idosos), enquanto em 2018 essa proporção era de 9,2\% (19,2 milhões) (ONU, 2014; SILVA et al., 2020).

A Organização Municipal de Saúde (OMS) descreve que a prevenção de quedas na velhice, estejam relacionados a doenças crônicas como: hipertensão, diabetes, artrose, artrite entre outras. $\mathrm{O}$ estudo comenta que o tratamento das quedas tende a aumentar nas próximas décadas com o crescimento da população idosa. Assim sendo, se faz necessários políticas de saúde que venha funcionar junto as necessidades da população idosa. A Estratégia Saúde da Família (ESF), está inserida no Sistema Único de Saúde (SUS), juntas tentam buscar estratégias para a prevenção das quedas na população idosa (SILVA et al., 2020).

O envelhecimento, é um processo natural que qualquer ser vivo estará suscetível, é acompanhado de inúmeros fatores biológicos, sociais e ambientais, que podem trazer consequências prazerosas ou angustiantes, sabemos o quanto nosso corpo pode sofrer com este processo, principalmente com patologias que ocorrem com mais facilidade a partir desta certa idade da vida, porém acompanhado de um estilo de vida saudável estas patologias podem ser controladas e até mesmo findadas (IBGE, 2016).

As equipes de saúde da família têm um papel primordial que é resgatar o vínculo dos profissionais/família, na busca de contribuir para melhoria da qualidade de saúde e de vida do indivíduo no ambiente familiar. Essas atribuições, devem ser executadas por meio de ações de assistência básica de vigilância epidemiológica e sanitária à criança, ao adolescente, à mulher, ao trabalhador e à terceira idade, enfim à população. O profissional de enfermagem tem papel relevante na equipe multidisciplinar (GOMIDE et al., 2018).

A Política Nacional de Atenção Básica (PNAB) desenvolve um conjunto de ações de saúde que visa à promoção e à proteção, à prevenção de agravos, ao diagnóstico, ao tratamento, a reabilitação e a manutenção da saúde. Entretanto, atenção à saúde da população idosa terá como porta de entrada a APS, tendo como referência a rede de serviços especializados APS e a ESF, surgiu para reorganizar os níveis de atenção, composta por uma equipe multiprofissional contendo médico, enfermeiro, técnico de enfermagem e agentes 
comunitários de saúde (BARRA et al., 2020).

A equipe de saúde da família atua com território adstrito, sendo responsável pela atenção à saúde dos indivíduos da sua área de abrangência. O fisioterapeuta insere-se na APS por meio dos Núcleos de Apoio à Saúde da Família (NASF). O NASF é composto por uma equipe de profissionais de diversas áreas (nutricionista, fisioterapeuta, psicólogo, assistente social, entre outros), tais profissionais realizam apoio para as ESF, possibilitando a ampliação das ações em saúde e qualificação da atenção (DEL ANTONI; ROCHA; CHIRELLI, 2017).

O fisioterapeuta, trabalha junto com a equipe multidisciplinar na prevenção de agravos, promoção da saúde e recuperação da saúde dos principais problemas de saúde dos idosos. Entre a quedas passou a serem consideradas umas principais causas de morbidade e mortalidade entre idosos, ela tem uma ligação direta com a postura e o equilíbrio trazendo consequências irreversíveis caso ocorra um tombo, existem vários fatores que podem ajudar o ocasionalmente da mesma após acontecimento da primeira queda o corpo de um idoso passa a se modificar se tornando mais frágil e mais fraco mudando completamente (GOMES et al., 2014).

A queda em idosos tem aumentado os índices principalmente em residências e locais públicos trazendo consequência lastimáveis a queda é considerada uma síndrome geriátrica preocupante, idosos por ser uma população um tanto desvalorizada e alguns muito fracos e com problemas tanto de saúde como psicológica é uma população que merece uma atenção de maior qualidade, por estar no fim de sua vida e ter contribuído bastante quando estava em atividade. A maioria da população idosa já possui problema nas articulações alguns deles sendo causados pela própria queda levando a um caso muitas das vezes irreversível.

Uma vez depois de ocorrida, a queda leva o paciente a sentir dores constantes no local causando incapacidade de se locomover e até mesmo fraturas e até problemas crônicos o cuidado e a prevenção devem ser feitos bem antes de ocorrer a primeira queda, a importância de o tema ser levado a público é fundamental. Palestras e conversas sobre o assunto é algo que necessita ter uma discussão e precursão maior, a matéria deve ser abordada para que seja estudada mais a fundo e que descubra os motivos de tantas quedas para que depois de ter uma causa formada advertir os idosos para que o índice de quedas diminua. Já que depois de certa idade os idosos merecem ter envelhecimento mais tranquilo e saudável. 
Este estudo pretende descrever a forma de atuação do fisioterapeuta na atenção básica em saúde, bem como refletir sobre a prevenção de quedas em idosos no contexto da atenção primária em saúde. 


\section{METODOLOGIA}

Trata-se de uma pesquisa de cunho exploratório, do tipo revisão integrativa da literatura. Por se tratar de pesquisa de cunho revisional, foram utilizadas as bases de dados eletrônicos por meio da Biblioteca Virtual em Saúde (BVS), aonde encontram-se outras bases de dados e periódicos inseridas, no entanto o presente trabalho usará as seguintes bases para estudo: Literatura Latino Americana e do Caribe em Ciências da Saúde (LILACS), Scientific Electronic Library Online (SCIELO), Biblioteca Virtual em Saúde Enfermagem Brasil (BDENF), PUBMED. Os critérios de inclusão da pesquisa foram: artigos que contemplem a temática de acordo com os descritores pesquisados, artigos no idioma português e inglês, artigos que possuam título, local de pesquisa, editora, ano de publicação. Como critérios de exclusão da pesquisa retiramos: pesquisas não originais, pesquisas não completas, com apenas o resumo ou faltando a parte de resultados e discussão.

O período de pesquisa de tais artigos se deu no período de janeiro a setembro de 2020 . Os descritores em saúde utilizados na pesquisa são: Atenção primária à saúde; Fisioterapia; Queda; Idosos. Por se tratar de uma revisão bibliográfica, o projeto não será submetido ao CEP conforme resolução número 466/12. Entretanto haverá o comprometimento de citar nomes dos autores utilizados no estudo, respeitando a norma regulamentadora (NR60023). Os dados foram utilizados exclusivamente com finalidade científica. 


\section{RESULTADOS}

Foram analisados 89 artigos publicados entre 2014a 2020. Com a estratégia de cruzamento: atenção primária em saúde; fisioterapia; queda; idosos. Após uma seleção criteriosa dos resumos, foram selecionados 10 estudos referentes ao tema do projeto.

Quadro - Analise dos artigos sobre atuação do fisioterapeuta nas prevenções de quedas em idosos no período de $2014-2020$.

\begin{tabular}{|c|c|c|c|c|}
\hline AUTOR/ANO & TITULO & METODOLOGIA & RESULTADO & CONCLUSÃO \\
\hline $\begin{array}{l}\text { CADER et al., } \\
2014 .\end{array}$ & $\begin{array}{ll}\text { Intervenção } & \\
\text { fisioterápica } & \text { e } \\
\text { prevenção } \quad \text { de } \\
\text { quedas } & \mathrm{em} \\
\text { idosos } & \end{array}$ & $\begin{array}{ll}\text { Revisão } & \text { da } \\
\text { Literatura } & \end{array}$ & $\begin{array}{l}\text { resultado a mudança de } \\
\text { posição do indivíduo } \\
\text { para um nível mais baixo } \\
\text { em relação a sua posição } \\
\text { inicial. Pessoas de todas } \\
\text { as idades apresentam } \\
\text { risco de sofrer queda. } \\
\text { Porém, para os idosos, } \\
\text { elas possuem um } \\
\text { significado muito } \\
\text { relevante, pois podem } \\
\text { levá-los à incapacidade, } \\
\text { injúria e morte. Seu custo } \\
\text { social é imenso e torna- } \\
\text { se maior quando o idoso } \\
\text { tem diminuição da } \\
\text { autonomia e da } \\
\text { independência ou passa a } \\
\text { necessitar diaca de } \\
\text { institucionalizacão. }\end{array}$ & $\begin{array}{l}\text { Os casos em que a } \\
\text { queda está associada } \\
\text { variam muito: afecções } \\
\text { osteomusculares, } \\
\text { neurológicas } \\
\text { cardiovasculares que } \\
\text { podem estar } \\
\text { representadas em um } \\
\text { grupo de pessoas ou até } \\
\text { num mesmo paciente. A } \\
\text { combinação e a } \\
\text { interligação } \\
\text { problemas médicos, } \\
\text { psicológicos, } \\
\text { reabilitativos } \\
\text { socioeconômicos } \\
\text { requerem a atenção do } \\
\text { fisioterapeuta }\end{array}$ \\
\hline $\begin{array}{l}\text { OLIVEIRA et } \\
\text { al., } 2017 .\end{array}$ & $\begin{array}{lr}\text { Fisioterapia } & \text { na } \\
\text { Prevenção } & \text { de } \\
\text { Quedas } & \mathrm{em} \\
\text { Idosos: } & \\
\text { Revisão } & \mathrm{de} \\
\text { Literatura } & \end{array}$ & $\begin{array}{l}\text { Revisão } \\
\text { Sistemática da } \\
\text { Literatura }\end{array}$ & 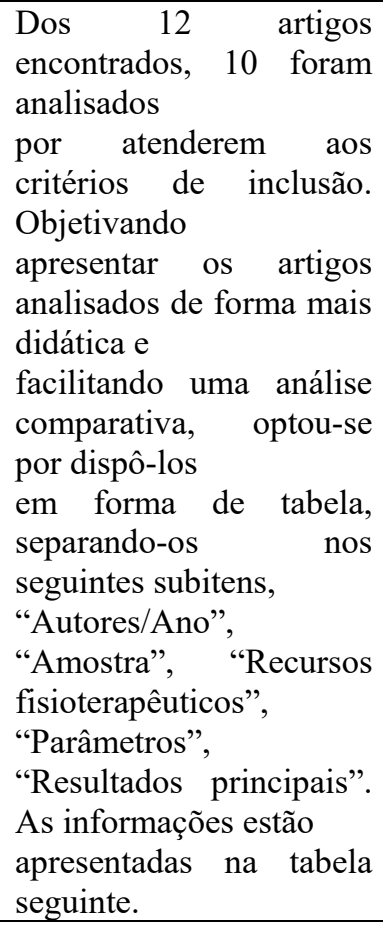 & $\begin{array}{l}\text { Conclui que a prática } \\
\text { regular de exercícios } \\
\text { físicos promove } \\
\text { melhoras significativas } \\
\text { nos aspectos equilíbrio, } \\
\text { flexibilidade, } \\
\text { funcionalidade e } \\
\text { aumento da resistência } \\
\text { muscular, reduzindo o } \\
\text { risco de quedas e } \\
\text { consequentemente } \\
\text { quebrando o ciclo } \\
\text { vicioso de quedas. }\end{array}$ \\
\hline $\begin{array}{l}\text { MATSUMOTO; } \\
\text { MILAGRES; } \\
\text { OMETTO- }\end{array}$ & $\begin{array}{l}\text { Centro } \\
\text { Universitário } \\
\text { Hermínio. }\end{array}$ & Estudo descritivo & $\begin{array}{l}\text { A pesquisa pode } \\
\text { identificar as } \\
\text { ações multidisciplinares }\end{array}$ & $\begin{array}{l}\text { Conclusão é a de que na } \\
\text { atenção ao idoso há } \\
\text { necessidade da atuação }\end{array}$ \\
\hline
\end{tabular}




\begin{tabular}{|c|c|c|c|c|}
\hline $\begin{array}{l}\text { UNIARARAS, } \\
2018 .\end{array}$ & $\begin{array}{l}\text { Atuação da } \\
\text { equipe } \\
\text { multidisciplinar } \\
\text { na prevenção } \\
\text { de quedas em } \\
\text { idoso } \\
\text { domicílio no }\end{array}$ & & $\begin{array}{l}\text { como propostas } \\
\text { preventivas, } \\
\text { visando a segurança do } \\
\text { paciente, minimizar o } \\
\text { risco de } \\
\text { quedas e melhorar a } \\
\text { qualidade de vida dos } \\
\text { idosos. }\end{array}$ & $\begin{array}{l}\text { de uma equipe } \\
\text { multiprofissional na } \\
\text { realização de um plano } \\
\text { de ação compartilhado, } \\
\text { com objetivo de definir } \\
\text { medidas de promoção } \\
\text { da saúde e prevenção de } \\
\text { agravos, decrescendo o } \\
\text { número de quedas em } \\
\text { idosos. }\end{array}$ \\
\hline $\begin{array}{l}\text { ILHA et a., } \\
2014 .\end{array}$ & $\begin{array}{l}\text { Quedas } \text { em } \\
\text { idosos: } \\
\text { reflexão para } \\
\text { os enfermeiros } \\
\text { e demais } \\
\text { profissionais }\end{array}$ & Estudo descritivo & $\begin{array}{lr}\text { Emergiram } & \text { três } \\
\text { categorias: } & \text { Alterações } \\
\text { relacionadas } & \text { ao } \\
\text { envelhecimento } \\
\text { facilitadoras de quedas } \\
\text { em idosos; principais } \\
\text { consequências das } \\
\text { quedas em idoso e } \\
\text { atuação do enfermeiro na } \\
\text { prevenção de quedas no } \\
\text { domicílio. }\end{array}$ & $\begin{array}{l}\text { Há necessidade } \\
\text { de } \text { desenvolver } \\
\text { estratégias r de } \\
\text { intervenção r com } \\
\text { objetivo de modificar a } \\
\text { configuração atual que } \\
\text { propicia a } \\
\text { ocorrência de quedas } \\
\text { em idosos. Descritores: } \\
\text { Acidentes por quedas; } \\
\text { Idoso; Enfermagem. }\end{array}$ \\
\hline SOUSA, 2019. & $\begin{array}{lr}\text { A Atuação } & \text { da } \\
\text { Fisioterapia } & \text { na } \\
\text { Prevenção } & \text { de } \\
\text { Quedas } & \mathrm{em} \\
\text { Idosos } & \end{array}$ & $\begin{array}{l}\text { Revisão } \\
\text { literatura }\end{array}$ & $\begin{array}{l}\text { A fisioterapia } \\
\text { desempenha importante } \\
\text { papel na prevenção das } \\
\text { quedas, pois proporciona } \\
\text { melhora do quadro motor } \\
\text { e do equilíbrio, além de } \\
\text { fornecer orientações aos } \\
\text { pacientes e seus } \\
\text { cuidadores, eliminando } \\
\text { ou minimizando os } \\
\text { fatores de risco de } \\
\text { pacientes de terceira } \\
\text { idade. }\end{array}$ & $\begin{array}{l}\text { Conclui-se que o papel } \\
\text { do Fisioterapeuta é } \\
\text { extrema importância } \\
\text { para prevenir quedas em } \\
\text { idosos através } \\
\text { exercícios para } \\
\text { equilíbrio corporal e } \\
\text { fortalecimento } \\
\text { muscular. Orientando } \\
\text { sobre os riscos de } \\
\text { quedas presentes em } \\
\text { casa, na rua, em } \\
\text { calçadas e em lugares } \\
\text { do seu cotidiano. }\end{array}$ \\
\hline $\begin{array}{l}\text { RESENDE- } \\
\text { NETO, } 2016 .\end{array}$ & $\begin{array}{l}\text { Treinamento } \\
\text { funcional para } \\
\text { idosos: uma } \\
\text { breve revisão }\end{array}$ & $\begin{array}{l}\text { Revisão } \\
\text { literatura }\end{array}$ & $\begin{array}{lr}\text { Os } & \text { resultados } \\
\text { encontrados sugerem que } \\
\text { o treinamento funcional } \\
\text { pode ser uma estratégia } \\
\text { eficaz e segura para } \\
\text { provocar respostas } \\
\text { adaptativas relacionadas } \\
\text { à funcionalidade do } \\
\text { idoso. }\end{array}$ & $\begin{array}{l}\text { Com base nos } \\
\text { resultados dos estudos } \\
\text { revisados, o treinamento } \\
\text { funcional parece ser } \\
\text { uma alternativa de } \\
\text { treinamento físico } \\
\text { segura, de baixo custo e } \\
\text { bastante interessante } \\
\text { para idosos, com } \\
\text { impacto positivo sobre a } \\
\text { massa muscular, força e } \\
\text { potência muscular, } \\
\text { resistência } \\
\text { cardiorrespiratória, } \\
\text { flexibilidade, equilíbrio } \\
\text { e cognição, podendo ser } \\
\text { implementada em } \\
\text { programas de promoção } \\
\text { de saúde nessa } \\
\text { população, } \\
\text { especificamente. }\end{array}$ \\
\hline $\begin{array}{l}\text { NEVES et al., } \\
2018 \text {. }\end{array}$ & $\begin{array}{l}\text { Fatores de risco } \\
\text { relacionados à } \\
\text { queda entre } \\
\text { idosos em uma }\end{array}$ & $\begin{array}{l}\text { Pesquisa de corte } \\
\text { transversal }\end{array}$ & $\begin{array}{l}\text { Os resultados mostraram } \\
\text { que a prevalência de } \\
\text { quedas relatadas pelos } \\
\text { idosos }\end{array}$ & $\begin{array}{l}\text { Conclui-se que a } \\
\text { prevalência de quedas } \\
\text { entre os idosos é } \\
\text { significativa, }\end{array}$ \\
\hline
\end{tabular}




\begin{tabular}{|c|c|c|c|c|}
\hline & $\begin{array}{l}\text { instituição } \\
\text { pública de um } \\
\text { município do } \\
\text { estado de Goiás }\end{array}$ & & $\begin{array}{l}\text { institucionalizados foram } \\
\text { de } 58,3 \% \text { o fator de } \\
\text { risco intrínseco de maior } \\
\text { incidência foi } 100 \% \text { o } \\
\text { uso de medicamentos e, } \\
\text { extrínseco foi a falta da } \\
\text { barra de apoio com } \\
41,7 \% \text {. }\end{array}$ & $\begin{array}{lr}\text { confirmando o objetivo } \\
\text { da pesquisa e } \\
\text { surgindo a necessidade } \\
\text { de adequações para } \\
\text { proporcionar r um } \\
\text { ambiente mais reguro } \\
\text { aos idosos. }\end{array}$ \\
\hline $\begin{array}{l}\text { CUNHA; } \\
\text { PINHEIRO, } \\
2016 .\end{array}$ & $\begin{array}{l}\text { O papel do } \\
\text { exercício físico } \\
\text { na prevenção } \\
\text { das quedas nos } \\
\text { idosos: uma } \\
\text { revisão baseada } \\
\text { na evidência }\end{array}$ & $\begin{array}{l}\text { Revisões } \\
\text { sistemáticas }\end{array}$ & $\begin{array}{l}\text { Foram encontrados } 203 \\
\text { artigos. Apenas cinco } \\
\text { preencheram os critérios } \\
\text { de inclusão: três MA e } \\
\text { duas RS. Há evidência de } \\
\text { que os programas de } \\
\text { exercícios para } \\
\text { prevenção de quedas em } \\
\text { pessoas mais velhas não } \\
\text { só reduzem a taxa de } \\
\text { quedas, mas também } \\
\text { evitam lesões decorrentes } \\
\text { das mesmas. Muitos dos } \\
\text { fatores de risco para } \\
\text { quedas são corrigíveis } \\
\text { por programas de } \\
\text { exercícios bes } \\
\text { desenhados e parecem } \\
\text { igualmente eficazes se } \\
\text { praticados no domicílio. } \\
\text { O tipo de EF mais eficaz } \\
\text { na prevenção das quedas } \\
\text { nos idosos é o treino de } \\
\text { equilíbrio e os programas } \\
\text { de EF são mais eficazes } \\
\text { se prolongados no tempo } \\
\text { e de maior intensidade } \\
\text { (maior número de horas e } \\
\text { pelo menos duas vezes } \\
\text { por semana). }\end{array}$ & $\begin{array}{l}\text { De acordo com a } \\
\text { evidência disponível, os } \\
\text { programas de EF devem } \\
\text { ser recomendados } \\
\text { porque são eficazes na } \\
\text { diminuição do número e } \\
\text { risco de quedas nos } \\
\text { idosos (Força de } \\
\text { recomendação A). }\end{array}$ \\
\hline JÚNIOR, 2016. & $\begin{array}{l}\text { Influência de } \\
\text { exercícios de } \\
\text { força muscular } \\
\text { para prevenção } \\
\text { de quedas em } \\
\text { idosos com } \\
\text { baixa massa } \\
\text { muscular: uma } \\
\text { revisão de } \\
\text { literatura }\end{array}$ & $\begin{array}{l}\text { Revisões } \\
\text { sistemáticas, }\end{array}$ & $\begin{array}{lr}\text { Foram encontrados cinco } \\
\text { artigos que atenderam } \\
\text { aos critérios deste estudo. } \\
\text { Quatro estudos } \\
\text { demonstraram que } \\
\text { programas para } \\
\text { prevenção de quedas, por } \\
\text { meio do ganho de força } \\
\text { muscular, acarretaram } \\
\text { em melhora do equilíbrio } \\
\text { e redução no número de } \\
\text { quedas. Um estudo } \\
\text { demonstrou que não } \\
\text { houve redução no no no dos } \\
\text { número de quedas do } \\
\text { seus participantes, porém } \\
\text { houve melhora do } \\
\text { equilíbrio. }\end{array}$ & $\begin{array}{l}\text { Programas de } \\
\text { fortalecimento muscular } \\
\text { parecem ser eficazes } \\
\text { para melhora do } \\
\text { equilíbrio em idosos e } \\
\text { redução da ocorrência } \\
\text { de quedas. }\end{array}$ \\
\hline $\begin{array}{l}\text { PORTELLA; } \\
\text { LIMA, } 2018 .\end{array}$ & 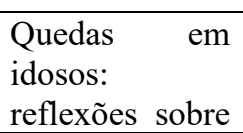 & Revisão Narrativa & \begin{tabular}{lcc}
\multicolumn{3}{l}{ Optou-se pelo método de } \\
revisão narrativa & por \\
entender que & são
\end{tabular} & $\begin{array}{ll}\text { Para OMS } & \text { trata-se de } \\
\text { uma } & \text { necessidade } \\
\text { urgente, } & \\
\end{array}$ \\
\hline
\end{tabular}




\begin{tabular}{|c|c|c|c|}
\hline $\begin{array}{l}\text { as políticas } \\
\text { públicas para o } \\
\text { envelhecimento } \\
\text { saudável }\end{array}$ & & $\begin{array}{l}\text { publicações amplas, } \\
\text { apropriadas para } \\
\text { descrever e discutir o } \\
\text { desenvolvimento ou o } \\
\text { "estado da arte" de um } \\
\text { determinado assunto, sob } \\
\text { o ponto de vista teórico } \\
\text { ou contextual, não } \\
\text { informando as fontes } \\
\text { utilizadas, a metodologia } \\
\text { para busca de } \\
\text { referências, nem os } \\
\text { critérios utilizados na } \\
\text { avaliação e de trabalhos. } \\
\text { seleção de } \\
\text { Para compor a revisão } \\
\text { delimitaram-se os } \\
\text { seguintes tópicos: } \\
\text { Envelhecimento como } \\
\text { fenômeno demográfico; } \\
\text { Envelhecimento e os } \\
\text { eventos de quedas; } \\
\text { inaugurar um novo } \\
\text { tempo de ação e de } \\
\text { parcerias, em cooperação } \\
\text { entre todos, pois se os } \\
\text { problemas são } \\
\text { multifacetados, como os } \\
\text { eventos de quedas. }\end{array}$ & $\begin{array}{l}\text { algo imprescindível que } \\
\text { pode ser feito em todos } \\
\text { os cenários, } \\
\text { não importa o nível de } \\
\text { desenvolvimento } \\
\text { socioeconômico. } \\
\text { Em termos de } \\
\text { prevenção de quedas, } \\
\text { cada uma das esferas } \\
\text { (união, estado e } \\
\text { municípios) devem } \\
\text { participar ativamente na } \\
\text { implementação de } \\
\text { programas e ações. }\end{array}$ \\
\hline
\end{tabular}

Fonte: próprio autor 


\section{DISCUSSÃO}

Para Cader et al. (2014), o fisioterapeuta proporciona um papel importante na prevenção de quedas por meio da orientação para a atividade física, alongamentos, fortalecimento muscular, treino de marcha e equilíbrio, visando a manutenção da capacidade funcional, proporcionando bem-estar. O exercício vem contribuindo na melhora e na saúde do idoso de forma geral, ajudando na prevenção de quedas, uma vez que oferece maior segurança na concretização das atividades básicas de vida, favorecendo o convívio social, melhora a saúde mental, mobilidade articular, autonomia e qualidade de vida.

Já oliveira et al. (2017), descreve que durante um diagnóstico preciso através de uma ferramenta de parâmetros clínicos preditores do risco de quedas em idosos, foram desenvolvidos diversos instrumentos para avaliação do controle postural. Entretanto, um desses instrumentos é conhecido como Timed Upand Go test (TUG), que tem proporcionado bons resultados como teste de equilíbrio envolvendo manobras funcionais, como levantasse, caminhar, dar uma volta e sentar-se. O TUG é um instrumento de triagem empregada com objetivo principal de avaliar a mobilidade e o equilíbrio.

Ainda assim, para que isso venha acontecer o fisioterapeuta necessitará de alguns instrumentos para realizar este exame físico do idoso. O relógio é cronometrado é essencial para cronometrar quando o paciente se levanta de uma cadeira (aproximadamente, 46 centímetros de altura), marcha em uma linha reta de 3 metros de distância (conforme seu ritmo que deverá ser confortável e seguro), vira, marcha de volta e senta-se sobre a cadeira novamente. Em um tempo menor para a execução deste trajeto desde o momento em que o idoso se encontra em repouso na cadeira indica um melhor desempenho funcional, enquanto a realização dessa atividade em um período maior indica maior risco de quedas (OLIVEIRA et al., 2017).

Segundo Cunha \& Pinheiro (2016), esses fatores estão relacionados ao ambiente devem ser identificados através da Escala Ambiental de Risco de Quedas, que contém itens que abordem a segurança de áreas de locomoção, disposição da mobília, iluminação, disponibilidade e acesso aos objetos, nos seguintes locais: quarto de dormir, banheiro, cozinha, escada e sala. O teste consta 29 itens, com escore menor de 10 pontos considerado de baixo risco, de 10 a 20 pontos médio risco e maior que 20 pontos, alto risco ambiental para quedas.

De acordo com Matsumoto, Milagres e Ometto-Uniararas (2018), avaliação do 
ambiente, é indispensável durante a orientação para reorganização do espaço interno, colocação de um diferenciador de degraus nas escadas, iluminação adequada, corrimão bilaterais para apoio, retirada de tapetes, instalação de pisos antiderrapantes, barras de apoio nos banheiros. Quanto às vestimentas adequadas, aconselha-se o uso sapatos com sola antiderrapante, amarrar o cadarço do calçado, trocar os chinelos que estão deformados.

Ilha et al. (2014), comenta que o fisioterapeuta vai atuar na habitação deste paciente para aplicar os testes de equilíbrio e escala ambiental e também na concretização de exercícios visando fortalecimento dos membros inferiores, resistência muscular, flexibilidade, equilíbrio e coordenação motora. Será realizado um planejamento incluindo os exercícios diários, adaptações necessárias e maior segurança do paciente em seu ambiente.

Já Sousa (2019), confirma que esses exercícios físicos são benéficos na prevenção de quedas nos idosos como intervenção única. Atividades de exercício que priorizam treinos de estabilização e práticas semanais com duração igual ou superior a 3 horas proporcionam melhores resultados. Para que isso venha acontecer é necessária uma manutenção nas atividades físicas moderada a intenso deve ser estimulado na prevenção de quedas a longo prazo.

Para Resende-Neto et al. (2016) atividade física apropriado tem a finalidade de aperfeiçoar o equilíbrio, da força muscular de membros inferiores e superiores e flexibilidade do ombro, contribuindo para a redução significativa do número de quedas entre os idosos, porém, não satisfatória para melhorar a marcha, flexibilidade multiarticular da coluna e quadril. Esses exercícios físicos são capazes diminuir o risco de quedas, favorecem uma melhora do equilíbrio e força muscular, que por sua vez são estratégias mais presentes nas intervenções em idosos.

Neves et al. (2016), comenta que os exercícios físico diariamente é importante para a preservação da funcionalidade, mas quando o idoso tem uma queda há obstáculo da autoconfiança, o que pode induzir ao medo de cair novamente, a insegurança, e com isso rejeição dos exercícios físico, acarretando redução da força muscular e consequentemente, um aumento do risco de quedas.

Cunha \& Pinheiro (2016), reforça que as quedas são as maiores causas de acidentes e de comprometimento da saúde das pessoas idosas, exercendo um sério problema de saúde pública. Constituindo a principal razão de acolhimento aos serviços de urgência neste grupo etário, assim como um dos principais fatores precipitantes da institucionalização desta 
população.

Júnior (2016), confirma que atividade física é capaz de amortecer ou prevenir o risco de quedas e umas das estratégias mais comuns são os alongamentos, exercícios resistência e equilíbrio. O motivo das quedas é multifatorial, e pode ser assinalado como uma alteração que pode ser normalizada ou prevenida. Para que o risco de quedas em idosos reduza, é preciso que os programas de saúde pública junto com de atividades físicas venha reforçar a força muscular seja normalizada ou recuperada, o equilíbrio seja restaurado e que ocorra uma diminuição do uso de medicamentos desses idosos.

Portella \& Lima (2018), relata que os benefícios da atividade físicas agem na melhora da capacidade funcional, equilíbrio, força, coordenação, velocidade de movimento e cognição, cooperando para uma maior segurança e prevenção de quedas entre as pessoas idosas. $\mathrm{O}$ fisioterapeuta é de extrema importância, porque permite uma melhora no equilíbrio integral, prevenindo quedas, tendo como inferência uma melhora na qualidade de vida desses indivíduos, aprimorando a força por meio de exercícios, de hidroterapia e orientações. 


\section{CONCLUSÃO}

Promover o envelhecimento saudável da população idosa é um dos grandes desafios da nossa sociedade, são vários fatores que desencadeiam o envelhecimento como sendo um fardo para muitos nessa fase da vida. As quedas são frequentes entre os idosos e produzem complicações que alteram negativamente a qualidade de vida dessas pessoas, afetando tanto fisicamente como psicologicamente, levando muitos a paralisarem suas atividades rotineiras e também a manter o isolamento no meio social.

O tratamento fisioterapêutico pode ser necessário em qualquer fase da vida, porém os idosos terá uma relevância não só de clínica, mas também de prevenção e intervenção, o que ajuda na melhora da qualidade de vida. Viabilizar a saúde junto aos idosos deve tornar-se um habito constante, por isso, é de suma importância o acompanhamento de um profissional qualificado atuando na terceira idade, promovendo praticas preventivas de exercícios físicos.

Com os exercícios de fortalecimento, de equilíbrio e de marcha, ambos trabalhados em sessões de fisioterapia, as quais levam o aumento da força muscular; melhora o equilíbrio, a flexibilidade, a coordenação motora e a propriocepção, além do ensinamento de exercícios que futuramente poderão ser realizados sozinhos pelo paciente, tornando assim uma vida saudável e prazerosa. Diante desse contexto a atuação do fisioterapeuta na prevenção a quedas durante a terceira idade é extremante importante no ambiente domiciliar. 


\section{REFERÊNCIAS}

BARRA, Jhayne Fonda et al. Avaliação da qualidade dos serviços de Atenção Primária à Saúde na perspectiva de enfermeiros. Research, Society and Development, v. 9, n. 10, p. e6319109075-e6319109075, 2020.

CADER, Samaria A. et al. Intervenção fisioterápica e prevenção de quedas em idosos. Revista HUPE, Rio de Janeiro. vol 13, n² 2, junho 2014.

CUNHA, Patricia; PINHEIRO, Costa Luisa. O papel do exercício físico na prevenção das quedas nos idosos: uma revisão baseada na evidência. Revista Portuguesa de medicina Geral e Familiar. Lisboa, vol 32, nº2, abril 2016.

DEL ANTONI, Ana Carolina Ferreira Tsunoda; DA ROCHA TONHOM, Silvia Franco; CHIRELLI, Mara Quaglio. Cuidado ao idoso na atenção básica: práticas de educação em saúde do fisioterapeuta. Revista Brasileira em Promoção da Saúde, v. 29, p. 5-15, 2017.

GOMES, Erika Carla Cavalcanti; MARQUES, Ana Paula Oliveira; LEAL, Marcia Corréra Campos; BARROS, Benvinda Pereira, Fatores associados ao risco de quedas em idosos institucionalizados: uma revisão integrativa. Ciência e saúde coletiva, p. 3543-3551,2014.

GOMIDE, Mariana Figueiredo Souza et al. User satisfaction with primary health care: an analysis of access and care. Interface-Comunicação, Saúde, Educação. v. 22, n. 65, p. 387398, 2018.

IBGE, Instituto Brasileiro de Geografia e Estatística. Síntese de Indicadores Sociais (SIS): Uma análise das condições de vida da população brasileira. [Internet]. Brasília. DF: IBGE; 2016. Disponível em: <https://biblioteca.ibge.gov.br/visualizacao /livros/liv101629.pdf>. Acesso em: 23.10.2020.

ILHA, Silomar et al. Quedas em idosos: reflexão para os enfermeiros e demais profissionais. Revista de Enfermagem UFPE. Recife, vol 8, junho 2014.

JÚNIOR, Adair Luiz. Influência de exercícios de força muscular para prevenção de quedas em idosos com baixa massa muscular: uma revisão de literatura. 2016.

MATSUMOTO, Viviane Santos; MILAGRES, Clarice Santana; OMETTO-UNIARARAS. Centro Universitário Hermínio. Atuação da equipe multidisciplinar na prevenção de quedas em idoso no domicílio. Revista Científica da FHO Uniararas, v. 6, n. 1, 2018.

NEVES, Ana et al. FATORES de risco relacionados à queda entre idosos em uma instituição pública de um município do estado de Goiás. 2016.

OLIVEIRA, D.V. et al. Comparison between selfreported functional capacity of brazilian and japanese institutionalized elderly. Rev. Pesq. Fisiot. v. 6, n. 3, p. 276-282, 2016.

OLIVEIRA, Hévelyn Moreira Lourenço et al. Fisioterapia na prevenção de quedas em idosos: revisão de literatura. Revista Interdisciplinar de Estudos Experimentais-Animais e Humanos Interdisciplinary Journal of Experimental Studies. v. 9, n. 1, 2017. 
ONU. Organização das Nações Unidas no Brasil. O mundo terá dois bilhões de idosos em 2050; OMS diz que 'envelhecer bem deve ser prioridade global'. Nov. 2014. Disponível em: $<\mathrm{http}$ ://nacoesunidas.org/mundo-tera-2-bilhoes-de-idosos-em-2050-oms-dizque-envelhecer-bem-deve-ser-prioridade-global/ >. Acesso em: 23.10.2020.

PORTELLA, Marilene Rodrigues; DE LIMA, Alisson Padilha. Quedas em idosos: reflexões sobre as políticas públicas para o envelhecimento saudável. Arquivos de Ciências da Saúde da UNIPAR, v. 22, n. 2, 2018.

RESENDE-NETO, Antônio Gomes et al. Treinamento funcional para idosos: uma breve revisão. Revista brasileira de ciência e movimento, v. 24, n. 3, p. 167-177, 2016.

SILVA, Joziane Santos et al. Ações na atenção básica para a prevenção de quedas em idosos/Nursingactions in basiccare for the prevention o ffalls in El derly. Brazilian Journal of Development, v. 6, n. 5, p. 22798-22808, 2020.

SOUSA, CREUZIMAR VIEIRA GOMES DE. A Atuação da Fisioterapia na Prevenção de Quedas em Idosos. 2019. 


\section{SOBRE OS AUTORES}

\section{Rutelene Oliveira Bailosa}

Acadêmica em Fisioterapia - Centro universitário do Norte

(UNINORTE), Pesquisadora Junior em Saúde Pública.

\section{Francisco Carlos Santos Cerqueira}

Docente em Fisioterapia - Centro universitário do Norte (UNINORTE) Manaus -AM. Doutorado em Ciências Militares. Escola de Comando e Estado Maior do Exército, ECEME, Brasil.
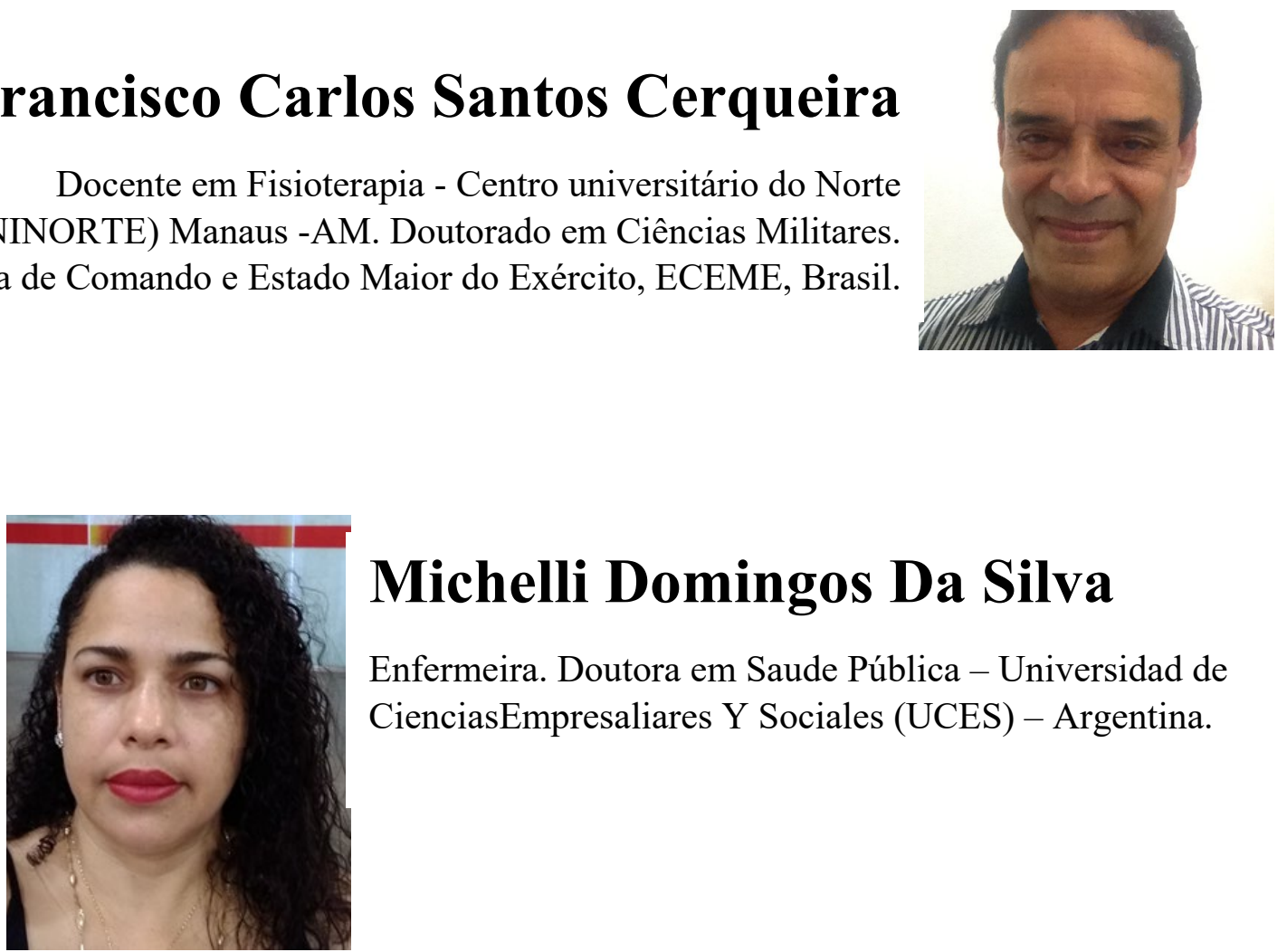

\section{Michelli Domingos Da Silva}

Enfermeira. Doutora em Saude Pública - Universidad de

CienciasEmpresaliares Y Sociales (UCES) - Argentina. 
www.editorapublicar.com.br contato@editorapublicar.com.br

@epublicar

facebook.com.br/epublicar

FISIOTERAPEUȚA NA ATENÇÃO PRIMARIA EM SAÚDE NO CONTEXTO DAS PREVENÇÕES DE QUEDAS EM IDOSOS:

\section{UMA REVISÃO INTEGRATIVA}

Rutelene Oliveira Bailosa Francisco Carlos Santos Cerqueira 
www.editorapublicar.com.br contato@editorapublicar.com.br

@epublicar

facebook.com.br/epublicar

FISIOTERAPEUȚA NA ATENÇÃO PRIMARIA EM SAÚDE NO CONTEXTO DAS PREVENÇÕES DE QUEDAS EM IDOSOS:

\section{UMA REVISÃO INTEGRATIVA}

Rutelene Oliveira Bailosa Francisco Carlos Santos Cerqueira 